\title{
The genetics of addiction
}

\author{
Nora D. Volkow $\cdot$ Maximilian Muenke
}

Published online: 5 May 2012

(C) Springer-Verlag (outside the USA) 2012

At the end of their report on the successful selective breeding of rat strains with either high or low susceptibilities to morphine addiction, Nichols and Hsiao (1967) conjecture that, although "we have not yet isolated the factors responsible for the difference in addiction liability, one factor seems to be passed on from generation to generation." That quaintly optimistic notion of "one factor" notwithstanding, this seminal study offers one of the earliest pieces of experimental evidence for the hereditary nature of addiction. Forty years later, and thanks to the findings from epidemiology and genetics studies, the general concept that addiction "runs in families" is beyond dispute, but teasing apart the timing, strength and contingent nature of the genetic contribution to addiction remains the focus of challenging research.

It is typically difficult to establish causal relationships between genetic/genomic variation and the risk of suffering from complex diseases. The factors that contribute to this are many: multiple common gene variants with small effects, non-linear interactions between genetic variants (epistasis) and phenotype severity, complex networks of gene-gene interactions, and similar phenotypes arising from distinct gene variants, just to name a few. In the case of substance use disorders (SUD), the powerful modulatory

N. D. Volkow $(\square)$

National Institute on Drug Abuse, National Human Research Institute, National Institutes of Health, U.S. Department of Health and Human Services, Rockville,

MD 20892-9581, USA

e-mail: nvolkow@nida.nih.gov

M. Muenke $(\bowtie)$

Medical Genetics Branch, National Human Genome Research Institute, National Institutes of Health, U.S. Department of Health and Human Services, Bethesda, MD, USA

e-mail: mamuenke@mail.nih.gov role played by complex environmental factors on brain processes, which further muddle the picture, is particularly relevant. This is because, in the absence of drug exposure, itself an environmental factor, the specific addiction phenotype would remain hidden, even in the presence of an overwhelming genetic load. On the other hand, brain development and architecture, which are partly determined by genetic factors, can be affected by exposure to drugs. These two-way interactions highlight the importance of genes involved in human brain development and function in the subsequent emergence of personality styles and of emotional and behavioral reactivities.

Indeed, multidisciplinary evidence reveals that the deceivingly simple concept of "genetics of addiction" belies the existence of a dense array of different interwoven layers whereupon genes, developmental processes, and environmental factors interact to increase or decrease the risk for SUD (Fig. 1a). In this special issue we have attempted to bring together different yet complementary research strategies whose ultimate goal is to better understand how genes contribute to SUD, including their effects on the various layers that determine whether and how the disease of addiction will be manifested (Fig. 1b). In this introduction we use the term addiction rather than the term "dependence" as described in DSM IV to avoid confusion with physical dependence, which is neurobiologically and clinically distinct from addiction. We refer to addiction as the phenotype characterized by the compulsive administration of the drug and the loss of control over its intake despite its adverse consequences to the individual. Note that, while relevant, we have not included a paper on the role of genes in brain development; readers interested in this topic are referred to various published reviews in the literature (Toga and Thompson 2005; Hariri 2009; Jedema et al. 2010; Mochida and Walsh 2004). 
A

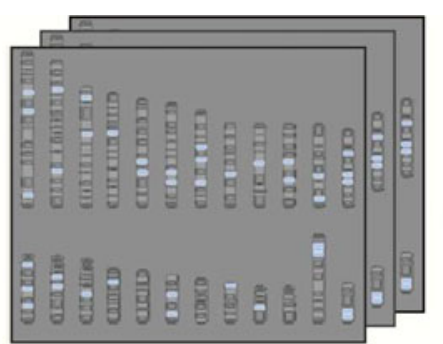

Genetic load

B
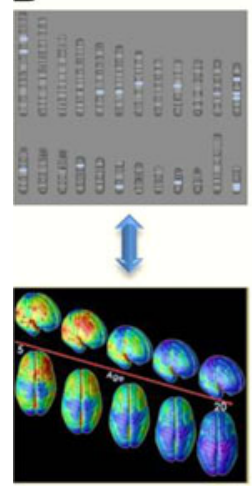

Brain

Development
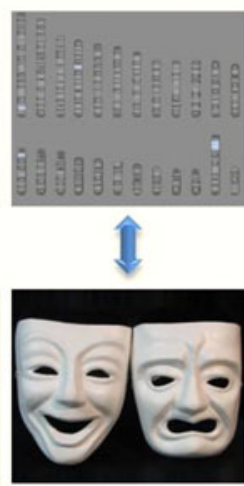

Personality

Comorbidities
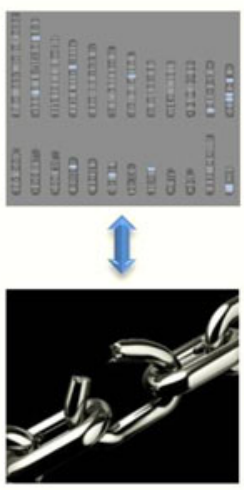

Stress-sensitivity Resiliency
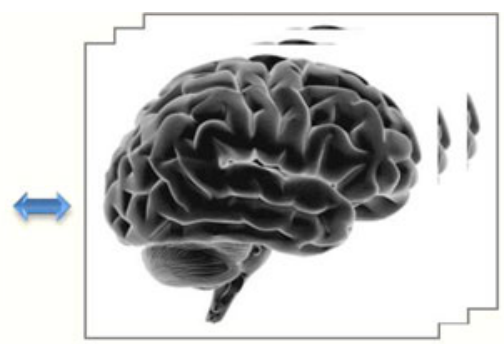

Brain outcomes
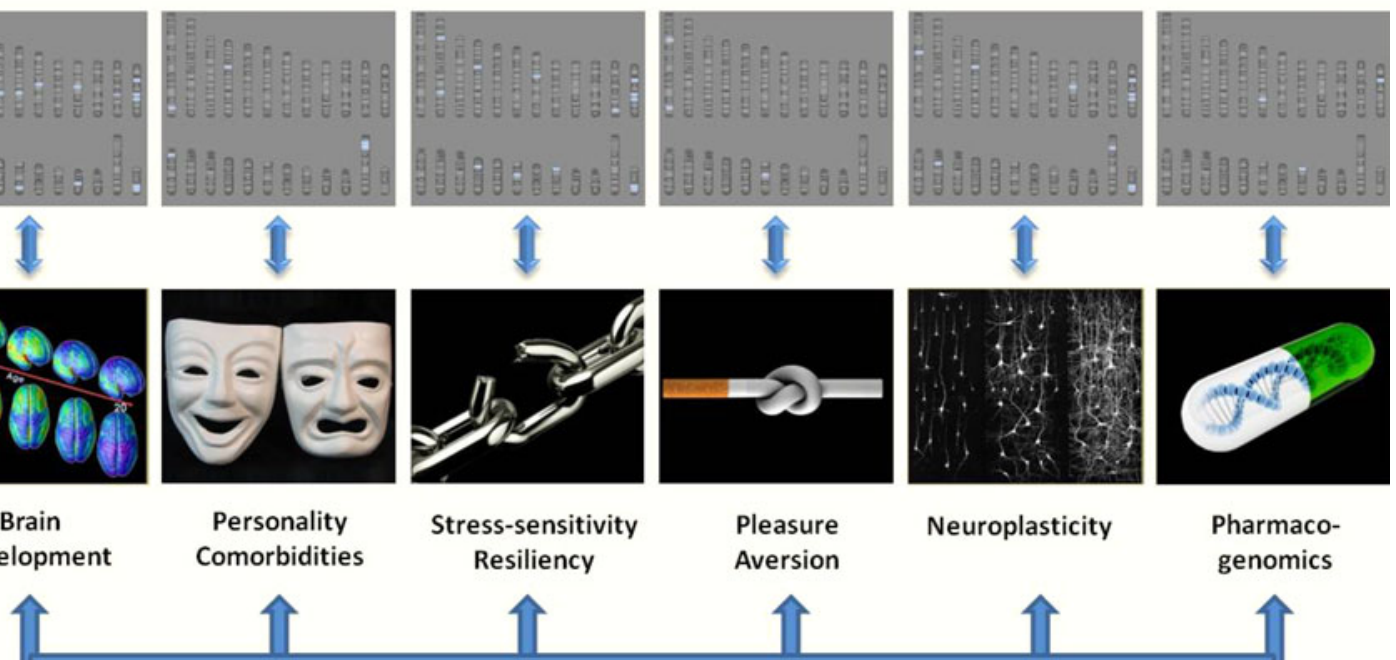

Pleasure

Aversion

Neuroplasticity

Pharmaco-

genomics
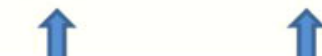

Fig. 1 The genetics and genomics of addiction. a There is significant evidence that genes contribute to close to half of the risk of suffering a substance use disorder (Volkow and Li 2005). But this robust relationship is far from being a univocal reflection between genetic variations on one hand and adverse drug-related behaviors on the other. b Rather, the powerful role of genetics reflects the combined

In this issue, the first paper, by Merikangas and McClair (2012), summarizes the epidemiological strategies used to characterize the prevalence of SUD and the estimates of its heritability. In addition to classical twin studies, the use of adoption and migration studies has corroborated the crucial role not only of genes but of social environments in increasing or decreasing the risk of a SUD. Such studies, however, cannot yet address the critical mechanistic question about how genetic diversity influences the sensitivity to either adverse or protective environments vis á vis abuse and addiction trajectories.

In their paper in this issue, Vrieze et al. (2012) discuss studies that address the interaction between genetic variation and development in the context of nicotine addiction. Using an elegant longitudinal design, they evaluated the relative contribution of genes versus environment as individuals progress from early adolescence into young adulthood. Their study identifies developmentally associated changes in the differential impact of these factors, such that their study demonstrates that the environment plays a impact of factors that operate at many different phenomenological levels, and is mediated through the encoding of multiple developmental, physiological, and behavioral processes as well as their interactions with equally powerful environmental factors, including drug exposure. We thank Dr. Ruben Baler from the National Institute on Drug Abuse who generated this figure

greater role in smoking behavior during adolescence, while the impact of genetic factors increases as they transition into young adulthood.

The molecular targets of the various substances of abuse have been, for the most part, well characterized. This knowledge offers a unique opportunity for investigating how variations in these targets influence the responses to drugs of abuse. Since all substances of abuse (both legal and illegal) exert their rewarding effects by increasing dopamine (DA) in the nucleus accumbens-a central node in the brain reward circuit-genetic variations affecting the DA system have been a natural and major focus of such efforts. However, the specific pathways used by different substances of abuse to increase DA vary among drug classes. Thus, the tetrahydrocannabinol (THC) in marijuana takes advantage of the cannabinoid receptor type 1 (CB1), heroin acts through mu opioid receptors and nicotine through nicotinic receptors; alcohol affects the dopaminergic system via multiple targets, including GABA, $\mathrm{N}$-methyl-D-aspartate (NMDA), cannabinoid and serotonin 
receptors. Several contributions to this special issue reflect the importance of investigating how allelic variations in each of these targets, which, by virtue of their potential to modulate dopamine and other signaling pathways, have been the focus of many candidate gene studies in SUD.

In their paper in this issue, Gorwood et al. (2012) review the characteristics of genes that are highly relevant to understanding SUD risk, particularly those which influence endophenotypic traits, such as stress reactivity, novelty seeking, and behavioral disinhibition/impulsivity. They also discuss how the architecture of these genes may affect their chances of undergoing epigenetic modifications, which have emerged as a critical element for understanding how chronic drug exposure is connected to long lasting changes in DA (and other neurotransmitters) signaling and cognitive performance (Robison and Nestler 2011; DiNieri et al. 2011).

Opioid neurotransmission is also crucial for signaling in the brain's reward pathway and, while directly relevant to heroin addiction, this neurotransmission pathway also modulates the acute and chronic responses to other substances of abuse (e.g., alcohol, nicotine, cocaine, and others). In their paper in this issue, Levran et al. (2012) review the various genes implicated in opioid neurotransmission and their associations with SUD, including the response to therapeutic interventions in the treatment of alcoholism and heroin addiction.

Alcohol's ability to enhance GABAergic neurotransmission (GABA acts as the main inhibitory neurotransmitter) is considered to be central to its rewarding effects. However, GABA has also been implicated in the rewarding effects of other substances of abuse. Specifically, recent experiments have revealed neuroadaptations in the GABAergic system that appear to be critically involved in the development of nicotine addiction. Cui et al's. (2012, this issue) contribution discusses the role of genes involved in GABA in alcoholism and nicotine addiction.

The genes encoding the various subunits that make up the nicotine receptors (which modulate cholinergic neurotransmission), have also been associated with SUD (and specifically with nicotine addiction). Nicotine receptors in DA neurons modulate neuronal activity and are therefore likely to also influence vulnerability to other SUDs (e.g., alcoholism) as well as the frequent comorbidities related to nicotine addiction and addiction to alcohol and other drugs. Gold and Lerman (2012, this issue) discuss the role of nicotine receptors in the context of pharmacogenetics with respect to their role in both the disease process itself (whereby an individual's response to a drug plays a determining role in the emergence of the disease of addiction) as well as in modulating treatment responses to nicotine addiction. Indeed, it is not surprising that genes that encode enzymes that metabolize nicotine may be implicated in addiction (variants with low enzymatic activity appear to confer some level of protection) as well as response to treatment (e.g., nicotine replacement therapy). Incidentally, pharmacogenetic studies on the treatment of SUD have focused on nicotine, alcohol, and heroin addictions, which are the only addictions for which there are approved medications.

SUD afflicted individuals frequently present with a comorbid condition, either a co-occurring addiction to a different drug or another psychiatric disorder. This clinical reality points to the existence of common genetic factors underlying vulnerability to both comorbid conditions. The paper in this issue by de Leon and Diaz (2012) discusses how comorbid conditions can be harnessed to investigate the genetics of SUD (in this case the comorbidity of schizophrenia or mood disorders with nicotine addiction). Although nicotine addiction and schizophrenia demonstrate the highest comorbidity rates, other diseases, and particularly mood disorders, also exhibit very high prevalence rates of smoking. The letter to the editor by Lawrence et al. (2012) in this issue provides evidence that the comorbidity between nicotine addiction and depression is not driven only by depressed patients' higher rates of abusing other drugs concomitantly to nicotine, but also by the direct effects of nicotine itself. The paper in this issue by Tsuang et al. (2012) discusses the complex interactions that underlie the comorbidity between nicotine addiction and depression, which are confounded by the antidepressant effects of smoking (both through nicotine and through inhibition of MAO A by cigarette smoke). They also discuss the potential causality role that early exposure to nicotine may have in increasing the risk of depression.

Attention deficit hyperactivity disorder is another psychiatric disorder that is associated with a higher risk for SUD. Arcos-Burgos et al. (2012, this issue) discuss common genes that have been associated with ADHD and SUD as well as with other externalizing disorders that are frequently comorbid with ADHD and SUD. Interestingly, their studies have identified genes that are part of pathways involved in synaptic architecture and in neurotransmission that also happen to be useful in predicting responses to ADHD treatment.

Four articles in this Special Issue discuss and review the value of animal models to study the contribution of genetic variation to SUD risk. The paper by Palmer and de Wit (2012) provides examples of how findings from animal models (related to genes that affect drug responses) can be used to interrogate the human genome (in order to identify candidate genes for further validation studies) and how findings from Genome Wide Association Studies (GWAS) in SUD that uncover new genes with unknown function, can be used to explore their role in acute or chronic drug responses. Fowler and Kenny (2012) present how a diverse 
array of genetically modified mice can be used to investigate the role of specific genes in drug responses. These include conventional knockout and knockin mice, and transgenic rodents expressing either modified proteins (alternative function or human variant) or genes with no endogenous ligand (e.g., channel rhodopsin for optogenetic stimulation or designer receptors for temporal activation with designer drugs). Another animal model for studying the effects of genetic manipulations in drug responses is the fly; the paper by Kaun et al. (2012) discusses the value and contributions of this model for identifying genes that modulate the sensitivity to the pharmacological effects of drugs, including their rewarding effects. In another paper, Klee et al. (2012) discuss the potential value of the zebrafish for studying the role of genes in modulating acute and chronic responses to drugs of abuse, particularly in human genes that have zebrafish paralogues.

This special issue also includes the results from a GWAS of nicotine addiction in individuals of Asian descent (Yoon et al. 2012). This GWAS identified association with a gene (FRMD4A) involved in protein scaffolding that is essential for synaptic plasticity. Genes involved with neuroplasticity in SUD have been identified across studies of a wide variety of SUDs (Uhl et al. 2008) and are also likely to contribute to comorbidity (see Arcos-Burgos et al. 2012). Moreover, the persistent nature of the changes induced by drugs in the brain reward pathway (and other circuits), along with the chronicity of the addictive process, are known to be closely linked to pathological neuroplasticity (Van den Oever et al. 2012; Kalivas and Volkow 2011).

It is important to point out that, with the exception of genes involved in protection against alcohol or nicotine addictions, via their effects on drug metabolism, the risk of SUDs attributable to other classes of genes identified to date is very small. However, findings from genetic/genomic studies have been extremely successful at expanding our understanding of the neurobiology underlying drug addiction while, at the same time, helping us identify new targets for medication development. They are also the harbinger of an exciting future when genetic and genomic information will be used to optimize clinical trials of new addiction medications and enhance the delivery of health care through personalized SUD prevention and treatment interventions.

\section{References}

Arcos-Burgos M, Vélez JI, Solomon BD, Muenke M (2012) A common genetic network underlies substance use disorders and disruptive or externalizing disorders. Hum Genet 131:917-929. doi:10.1007/s00439-012-1164-4 (this issue)
Cui WY, Seneviratne C, Gu J, Li MD (2012) Genetics of GABAergic signaling in nicotine and alcohol dependence. Hum Genet 131:843-855. doi:10.1007/s00439-011-1108-4 (this issue)

de Leon J, Diaz FJ (2012) Genetics of schizophrenia and smoking: an approach to studying their comorbidity based on epidemiological findings. Hum Genet 131:877-901. doi:10.1007/s00439-0111122-6 (this issue)

DiNieri JA, Wang X, Szutorisz H, Spano SM, Kaur J, Casaccia P, Dow-Edwards D, Hurd YL (2011) Maternal cannabis use alters ventral striatal dopamine D2 gene regulation in the offspring. Biol Psychiatry 70:763-769

Fowler CD, Kenny PJ (2012) Utility of genetically modified mice for understanding the neurobiology of substance use disorders. Hum Genet 131:941-957. doi:10.1007/s00439-011-1129-z (this issue)

Gold AB, Lerman C (2012) Pharmacogenetics of smoking cessation: role of nicotine target and metabolism genes. Hum Genet 131:857-876. doi:10.1007/s00439-012-1143-9 (this issue)

Gorwood P, Le Strat Y, Ramoz N, Dubertret C, Moalic JM, Simonneau M (2012) Genetics of dopamine receptors and drug addiction. Hum Genet 131:803-822. doi:10.1007/s00439-0121145-7 (this issue)

Hariri AR (2009) The neurobiology of individual differences in complex behavioral traits. Annu Rev Neurosci 32:225-247

Jedema H, Gianaros PJ, Greer PJ, Kerr DD, Liu S, Higley JD, Suomi SJ, Olsen AS, Porter JN, Lopresti BJ, Hariri AR, Bradberry CW (2010) Cognitive impact of genetic variation of the serotonin transporter in primates is associated with differences in brain morphology rather than serotonin neurotransmission. Mol Psychiatry 15:12-22

Kalivas P, Volkow ND (2011) New medications for drug addiction hiding in glutamatergic neuroplasticity. Mol Psychiatry 16:974986

Kaun KR, Devineni AV, Heberlein U (2012) Drosophila melanogaster as a model to study drug addiction. Hum Genet 131:959-975. doi:10.1007/s00439-012-1146-6 (this issue)

Klee EW, Schneider H, Clark KJ, Cousin MA, Ebbert JO, Hooten WM, Karpyak VM, Warner DO, Ekker SC (2012) Zebrafish: a model for the study of addiction genetics. Hum Genet 131:977-1008. doi:10.1007/s00439-011-1128-0 (this issue)

Lawrence D, Mitrou F, Zubrick SR (2012) Premature to conclude no genetic basis to the association between smoking and major depressive disorder. Hum Genet 131:903-904. doi:10.1007/ s00439-012-1169-z (this issue)

Levran O, Yuferov V, Kreek MJ (2012) The genetics of the opioid system and specific drug addictions. Hum Genet 131:823-842. doi:10.1007/s00439-012-1172-4 (this issue)

Merikangas K, McClair V (2012) Epidemiology of substance use disorders. Hum Genet 131:779-789. doi:10.1007/s00439-0121168-0 (this issue)

Mochida G, Walsh CA (2004) Genetic basis of developmental malformations of the cerebral cortex. Arch Neurol 61:637-640

Nichols JR, Hsiao S (1967) Addiction liability of albino rats: breeding for quantitative differences in morphine drinking. Science 157:561-563

Palmer AA, de Wit H (2012) Translational genetic approaches to substance use disorders: bridging the gap between mice and humans. Hum Genet 131:931-939. doi:10.1007/s00439-0111123-5 (this issue)

Robison A, Nestler EJ (2011) Transcriptional and epigenetic mechanisms of addiction. Nat Rev Neurosci 12:623-637

Toga AW, Thompson PM (2005) Genetics of brain structure and intelligence. Annu Rev Neurosci 28:1-23

Tsuang M, Stone W, Francis T, Minor K, Thomas A (2012) Genetics of smoking and depression. Hum Genet 131:905-915. doi: $10.1007 / \mathrm{s} 00439-012-1170-6$ (this issue) 
Uhl G, Drgon T, Johnson C, Li CY, Contoreggi C, Hess J, Naiman D, Liu QR (2008) Molecular genetics of addiction and related heritable phenotypes: genome-wide association approaches identify "connectivity constellation" and drug target genes with pleiotropic effects. Ann N Y Acad Sci 1141:318-381

Van den Oever MC, Spijker S, Smit AB (2012) The synaptic pathology of drug addiction. Adv Exp Med Biol 970:469-491

Volkow N, Li TK (2005) The science of addiction. Nat Neurosci $8: 1429-14230$

Vrieze SI, McGue M, Iacono WG (2012) The interplay of genes and adolescent development in substance use disorders: leveraging findings from GWAS meta-analyses to test developmental hypotheses about nicotine consumption. Hum Genet 131:791801. doi:10.1007/s00439-012-1167-1 (this issue)

Yoon D, Kim Y-J, Cui W-Y, Van der Vaart A, Cho YS, Lee J-Y, Ma JZ, Payne TJ, Li MD, Park T (2012) Large-scale genome-wide association study of Asian population reveals genetic factors in FRMD4A and other loci influencing smoking initiation and nicotine dependence. Hum Genet 131:1009-1021. doi:10.1007/ s00439-011-1102-x (this issue) 